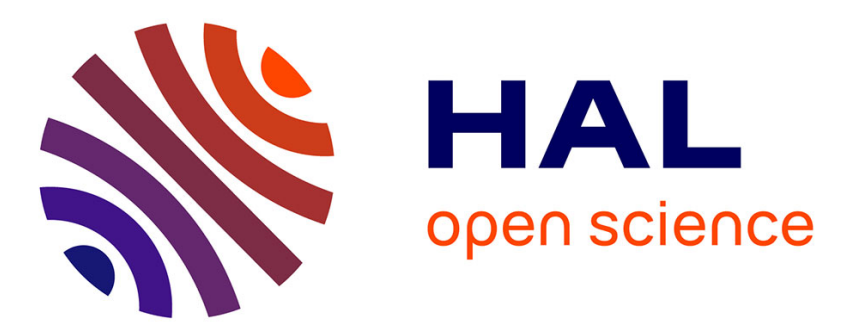

\title{
Growth of Clathrate Hydrates from Water Drops in Cyclopentane
}

Jorge Peixinho, Valentin Ageorges, Benoit Duchemin

\section{To cite this version:}

Jorge Peixinho, Valentin Ageorges, Benoit Duchemin. Growth of Clathrate Hydrates from Water Drops in Cyclopentane. Energy \& Fuels, 2018, 32 (3), pp.2693-2698. 10.1021/acs.energyfuels.7b02740 . hal-02143426

\section{HAL Id: hal-02143426 https://hal.science/hal-02143426}

Submitted on 29 May 2019

HAL is a multi-disciplinary open access archive for the deposit and dissemination of scientific research documents, whether they are published or not. The documents may come from teaching and research institutions in France or abroad, or from public or private research centers.
L'archive ouverte pluridisciplinaire HAL, est destinée au dépôt et à la diffusion de documents scientifiques de niveau recherche, publiés ou non, émanant des établissements d'enseignement et de recherche français ou étrangers, des laboratoires publics ou privés. 


\title{
Growth of Clathrate Hydrates from Water Drops in Cyclopentane
}

\author{
Jorge Peixinho, ${ }^{*}$ Valentin Ageorges, and Benoit Duchemin \\ Laboratoire Ondes et Milieux Complexes, CNRS et Université Le Havre Normandie, 76600 \\ Le Havre, France \\ E-mail: jorge.peixinho@univ-lehavre.fr
}

\begin{abstract}
Clathrate hydrates are icelike crystalline compounds with encaged guest molecules trapped inside the cages of hydrogen bonded water molecules. Their growth is visualized for cyclopentane within water drops of two microliters on glass and polytetrafluoroethylene surfaces. The effect of the interfacial tension between water and cyclopentane is measured at different temperatures and for different concentration of an oil-soluble surfactant: sorbitan monooleate (Span 80). The drops experience a temperature sequence where they freeze into ice, form hydrates and melt. Cyclopentane hydrates crystals are affected by the concentration of surfactant. The morphology seen here could be relevant for explaining the behavior of hydrate emulsions.
\end{abstract}

\section{Introduction}

Clathrate hydrates ${ }^{1}$ (abbreviated hydrates) are crystalline solids composed of water molecules called the "host" that form cages in which "guests" molecules are enclosed. The guests maybe light hydrocarbons, cyclopentane (abbreviated CP), carbon dioxide, methane, rare gas, etc. It is generally acknowledged that hydrates have properties that include a large capacity of 
gas storage, fractionation of gas mixtures, and a high heat of formation and decomposition. These properties enable hydrates to be used for various technologies in transportation and storage of natural gas, sequestration of carbon dioxide and even water desalination. ${ }^{2}$ There are several types of hydrates that differ in crystallographic cage structure: type I, type II and type $\mathrm{H}$, which nature and size depend on the guest molecule. CP guests ${ }^{1}$ often lead to the formation of type II.

Most of previous works were concerned with the kinetic of hydrates crystallization under different pressure and temperature conditions. ${ }^{3}$ Typically, hydrates are formed at temperatures below about $4^{\circ} \mathrm{C}(277 \mathrm{~K})$ in combination with elevated pressure in the range of $1-10$ MPa. However, not all hydrate-forming systems require elevated pressures and both CP and tetrahydrofuran (THF) form hydrates at atmospheric pressure and accessible temperatures. For example, the melting temperature of $\mathrm{CP}$ hydrate is approximately $7^{\circ} \mathrm{C}(280 \mathrm{~K})$ at atmospheric pressure. ${ }^{4}$ This allows the study of hydrate formation processes without the experimental difficulties of dealing with pressure. Since THF is nearly fully miscible with water, the essential issue of mass transfer of the hydrate former (guest species) from the external organic phase to the water drops is not present in a THF-water system, whereas CP-water systems retain an interface.

The growth of hydrates deserves to be studied because its implication in the blockage of pipelines, i.e. flow assurance. Previous investigations on hydrate suspensions in oil dominated systems, ${ }^{5}$ water-in-crude oil emulsions ${ }^{6}$ and black oil suspensions ${ }^{7}$ have tested the effects of temperature and conversion rate of water into hydrates. However, the detailed effect of each system's component is sometimes difficult to measure quantitatively. Hence, model systems of water-in-oil emulsions are used to mimic the hydrates growth. Specifically, the presence of hydrates is detected from the significant increase of the viscosity seen in rheological measurements during temperature quench. This overshoot can not be explained by the simple conversion of water drops into ice or hydrates and has been used to explore the effect of different parameters controlling the formation of hydrates for different sub-cooling 
temperatures,${ }^{8,9}$ shear rates, ${ }^{9-11}$ water fractions, ${ }^{8,9,11-13}$ surfactant concentrations, ${ }^{9}$ cooling rates ${ }^{12}$ and salt concentration. ${ }^{11}$ A mechanism based on random nucleation of water droplets into porous hydrate particle, ${ }^{14}$ agglomeration ${ }^{15}$ and capillary bridging ${ }^{16}$ has been proposed. Yet, the quantitative understanding of the interaction, between the hydrate particle roughness and the apparent volume fraction, remains to be explained.

In addition, $\mathrm{CP}$ hydrates formation have been observed in previous studied by Sakemoto et al., ${ }^{17}$ Ishida et al., ${ }^{18}$ Karanjkar et al., ${ }^{19}$ Mitarai et al. ${ }^{20}$ and recently by de Banos et al. ${ }^{21}$ All previous investigations indicate a growth in two stages: (i) nucleation at the interface and (ii) growth depending on the temperature, the presence of surfactant or properties of the wall surfaces. Our objective is to study a single water drop ${ }^{22,23}$ as a simplified model of an emulsion of water in $\mathrm{CP}$ with an oil-soluble surfactant: sorbitan monooleate (Span 80), test the results of Karanjkar et al. ${ }^{14,19}$ and add new data to understand the complex morphogenesis of this relatively simple system. Few studies are at the scale of a single drop presumably because the formation of a "halo", a hydrate crust growing simultaneously on the water drop and also radially on the glass substrate. ${ }^{21,24}$ Here, this difficulty is circumvented using polytetrafluoroethylene (PTFE) surface film to hold the drop. The effect of the influence of inhibitors and anti-agglomerants such as colloidal particles, polymer surfactants or salt ${ }^{17}$ has to be taken into account as their interfacial and adhesive properties are know to affect the kinetic of hydrates. ${ }^{25,26}$

This paper is composed of three parts. In the first part, the fluid used and the experimental setup are described together with new measurements of the fluid properties such as the interfacial tension as a function of temperature and surfactant concentration. In the second part, the growth and dissociation of CP-hydrates are observed and monitored for a well-defined temperature protocol. Finally, a summary of the experiments of CP-hydrates formation and dissociation times and temperatures for various surfactant concentrations is presented and discussed. 


\section{Materials and methods}

\section{Fluids}

Ultra pure water is drawn from a purification system, which produces water with a constant electric resistivity of $18.2 \mathrm{M} \Omega . \mathrm{cm}$. The oil phase is CP (reagent grade of $98 \%$ ). CP is a ring molecule, $\mathrm{C}_{5} \mathrm{H}_{10}$, with a density of 0.751 g. l $^{-1}$, a melting temperature of $-93.9^{\circ} \mathrm{C}$ $(179.25 \mathrm{~K})$ and a boiling temperature of $49.2^{\circ} \mathrm{C}(322.35 \mathrm{~K})$. Next, a water drop is placed in a bath of CP. At room temperature, CP is known to be weakly soluble in water (156 mg. $\left.1^{-1}\right)$. In most cases, CP is mixed with an oil-soluble surfactant: sorbitan monooleate or Span 80, used as received (from Sigma Aldrich). $\mathrm{C}_{24} \mathrm{H}_{44} \mathrm{O}_{6}$ is a non-ionic surfactant with an hydrophilic-lipophilic balance (HLB) of $4 \pm 1$. Other sorbitan monooleate surfactants (Span 20, 40 and 60) with different chain lengths have been shown to have an influence on the drop sizes and the dissociation temperature. ${ }^{27}$ In addition, heptane (from VWR Chemicals with a reagent grade of $98 \%$ ) was used as test case. The properties of the fluids depend on temperature. The densities of CP and water have been measured using a densimeter (DMA 35 from Anton Paar) with an accuracy of $10^{-3}$ g. $\mathrm{cm}^{-3}$ and are in good agreement with the previous results for alkanes ${ }^{28}$ and for water. ${ }^{29}$ The stoichiometric CP to water molar ratio for complete conversion to hydrate is about 1:17 according to Zhang et al. ${ }^{30}$ so $\mathrm{CP}$ is in excess for the hydrate formation reaction.

\section{Interfacial tension}

Interfacial tension was measured using a drop shape tensiometer (DSA100 manufactured by Krüss). The measure is based on the Young-Laplace model ${ }^{31}$ and the shape of the drop to calculate the interfacial tension, $\sigma$. The $\mathrm{CP}$ rising drops were formed using an inverted needle ( $\mathrm{J}$ type) of $2.13 \mathrm{~mm}$ outer diameter in water. The water temperature was controlled using a water bath (Julabo F12). Figure 1 presents the interfacial tension measurements for heptane and CP for different temperatures. The results for the interfacial tension of heptane 
in water are in agreement with Zeppieri et al. (2001). ${ }^{32}$ Errors bars have been added and represent a variance over 15 measurements. For $\mathrm{CP}$, the new data are consistent with the measurement of Karanjkar et al. ${ }^{19}$ The interfacial tension of pure fluids are relatively high and decrease with temperature is a linear fashion.

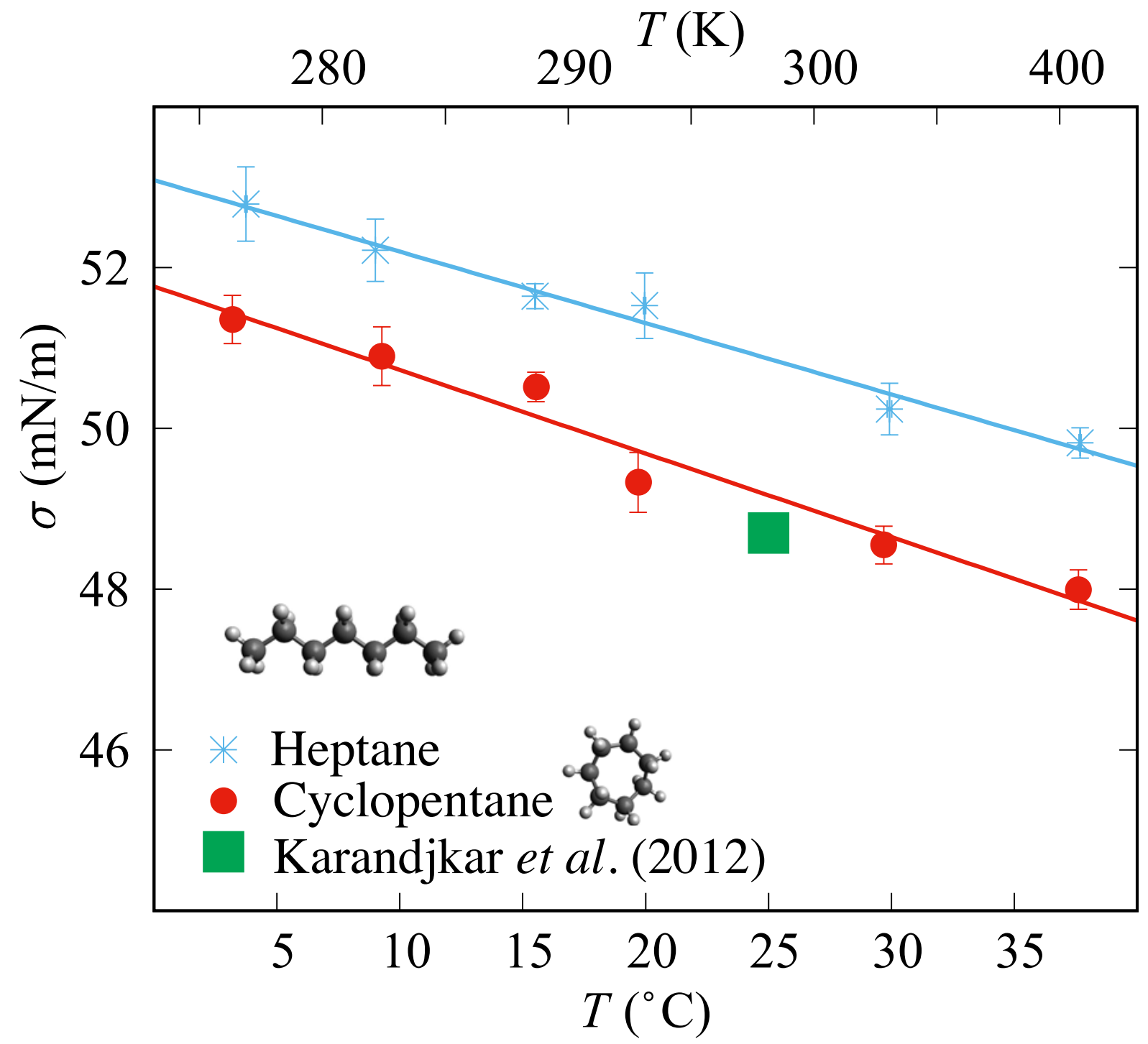

Figure 1: Interfacial tension, $\sigma$, versus temperature, $T$, for Heptane and cyclopentane (CP). The (green) square represents the data from Karanjkar et al. ${ }^{19}$ The lines are linear fits of the data.

In order to quantify the effects of Span 80 on the interfacial tension, mixtures of $\mathrm{CP}$ with six different concentration, $C_{\mathrm{S} 80}$, of Span 80 (in volume) have been prepared. Figure 2 
shows the equilibrium interfacial tension of water and $\mathrm{CP}$ as a function of $C_{\mathrm{S} 80}$ over several decades of concentration. Results are obtained for two temperatures: $3^{\circ} \mathrm{C}(276.15 \mathrm{~K})$ and $20^{\circ} \mathrm{C}(293.15 \mathrm{~K})$. The results of Karanjkar et al. ${ }^{19}$ are also reproduced in figure 2 and are lower but is reasonably good agreement the present data. The critical micelle concentration or $\mathrm{CMC}$ can be identified around $0.025 \%(\mathrm{v} / \mathrm{v})\left(\right.$ or $\left.6 \times 10^{-4} \mathrm{~mol} / \mathrm{l}\right)$, again in good agreement with results of Karanjkar et al. ${ }^{19}$ Below $\mathrm{CMC}$, the variation of the interfacial tension, $\sigma$, with $C_{\mathrm{S} 80}$, can be described by the Langmuir adsorption isotherm: ${ }^{33}$

$$
\frac{\Gamma_{e}}{\Gamma_{\infty}}=\frac{1}{1+\left(\frac{\alpha}{\beta C_{\mathrm{S} 80}}\right)},
$$

where $\Gamma_{e}$ is the equilibrium instantaneous surface concentration of Span $80, \Gamma_{\infty}$ is the surface concentration of Span $80\left(45 \AA^{2}\right.$ per adsorbed Span 80 molecule or $3.7 \times 10^{-6}$ mol.m $\left.{ }^{-2}\right)$ at saturation and $\alpha / \beta$ is the only adjustable parameter representing the kinetic rate constants obtained from least square methods.

Substituting $\Gamma_{e}$ by $\Gamma$ from equation (1) in the equation of state for the interfacial tension, the experimental data can be described by:

$$
\sigma(\Gamma)=\sigma_{c}+R T \Gamma_{\infty}\left[\ln \left(1-\frac{\Gamma}{\Gamma_{\infty}}\right)\right]
$$

where $\sigma_{c}$ represents the clean interfacial tension without Span 80, $R$ is the universal gas constant $\left(8.314 \mathrm{~J} . \mathrm{mol}^{-1} \cdot \mathrm{K}^{-1}\right)$ and $T$ is the temperature in Kelvin.

For large $C_{\mathrm{S} 80}$, the present measurements are below the values from Karanjkar et al. ${ }^{19}$ This could be explained by the sensitivity of low interfacial tension to tip contact angles. These delicate measurements could be better-measured using spinning drop tensiometer.

\section{Visualization}

A Linkam cell (model THMS600) is used that allows for temperature control through nitrogen gas coolant. It is fixed on a microscope (Olympus BX51). This microscope can be used in 


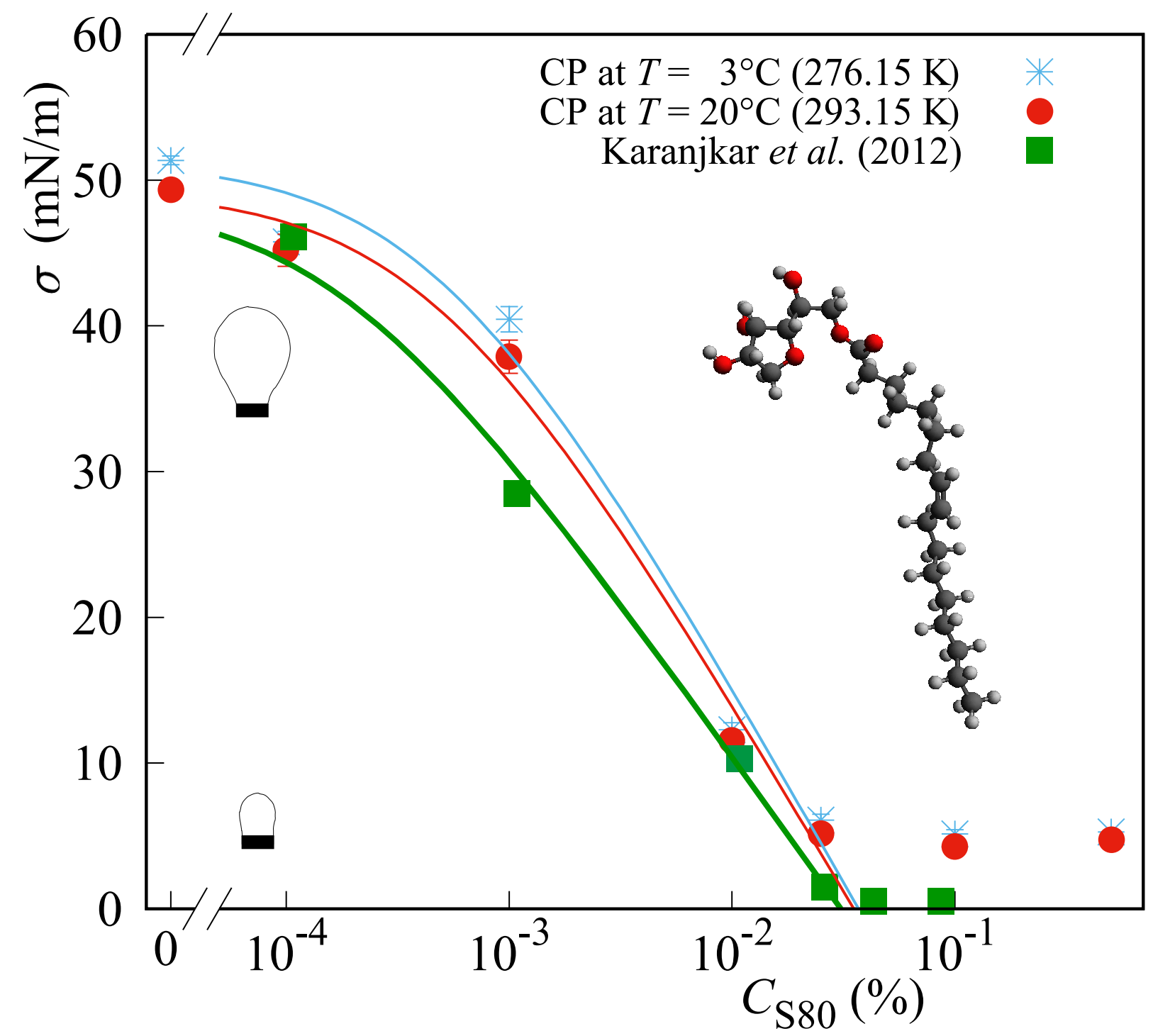

Figure 2: Interfacial tension; $\sigma$, versus Span 80 concentration, $C_{\mathrm{S} 80}(\mathrm{v} / \mathrm{v})$, for different temperatures. The lines are Langmuir fits for $C_{\mathrm{S} 80}<\mathrm{CMC}$. The (green) squares and line are results by Karanjkar et al. ${ }^{19}$ at $25^{\circ} \mathrm{C}(298.15 \mathrm{~K})$. Insets are drawings of the drop shapes (left) and the molecular structure of Span 80 (right). 


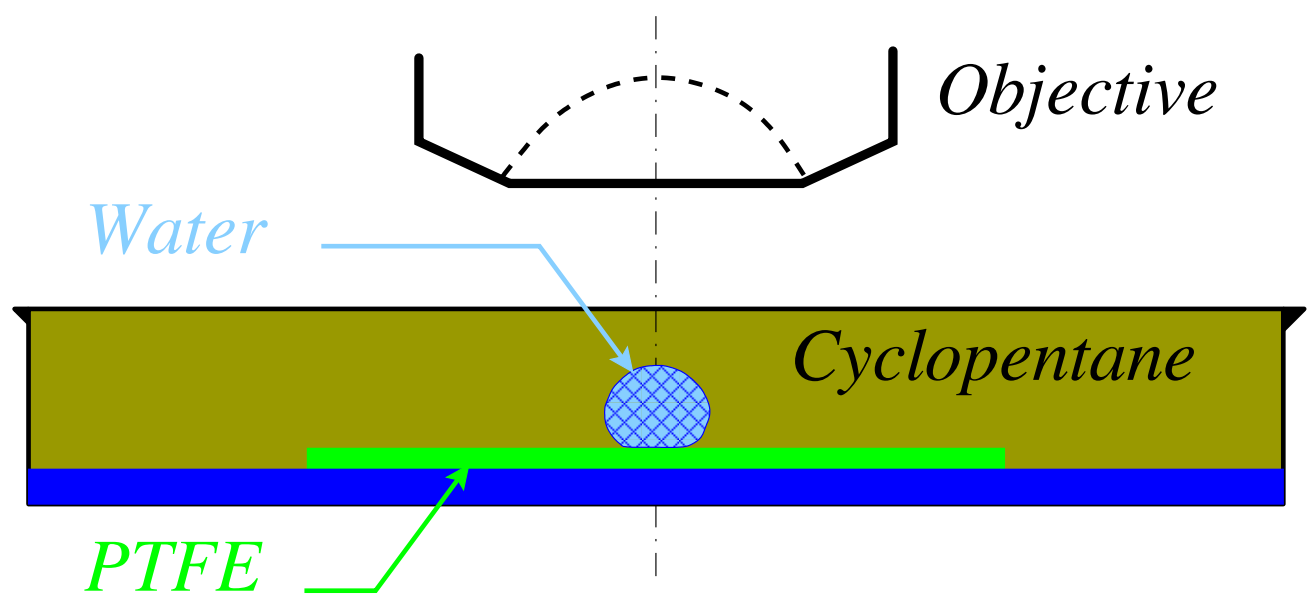

Figure 3: Schematic of the experimental setup. The container is filled with $\mathrm{CP}$ and a single $2 \mu \mathrm{l}$ water drop is formed on the PTFE surface. The temperature protocol is applied and the observations are from the top.

transmission or in reflection and with white polarized light. Focus $2.5 \times, 10 \times, 20 \times$ and $50 \times$ objectives were used to observe the drops. A schematic of the experimental setup is presented on figure 3. The diameter of the container is $16 \mathrm{~mm}$ and has a height of $2 \mathrm{~mm}$. The water drops were prepared using a micropipette (Ependorff) with a volume of $2 \pm 0.05 \mu$ l. The drop used here are slightly larger than the ones studied by Ning and Liu ${ }^{23}$ and of similar size than those by Karanjkar et al. ${ }^{19}$ Yet, the nucleation temperature of the water drop in $\mathrm{CP}$ is related to the drop diameter and the presence of impurities remains variable for every experiments and is in the range $-15^{\circ} \mathrm{C}(258 \mathrm{~K})$ to $-25^{\circ} \mathrm{C}(248 \mathrm{~K})$. The drop is placed on polytetrafluoroethylene (PTFE) disks in a bath of CP. It is pinned by a small laser cut hole that prevents the drop to move during the temperature protocol. Finally a cover slip is over the container.

\section{Temperature protocol}

In order to form hydrates, a temperature protocol ${ }^{19,34}$ is chosen that first converts the water into ice. In the present experiments, the presence of ice is used to trigger the formation of hydrates. The temperature ramping rate is fixed at $5^{\circ} / \mathrm{min}$ and is similar to the ramping rate of previous experiments. ${ }^{19,34}$ The temperature protocol is sketched in figure 4 and is as 


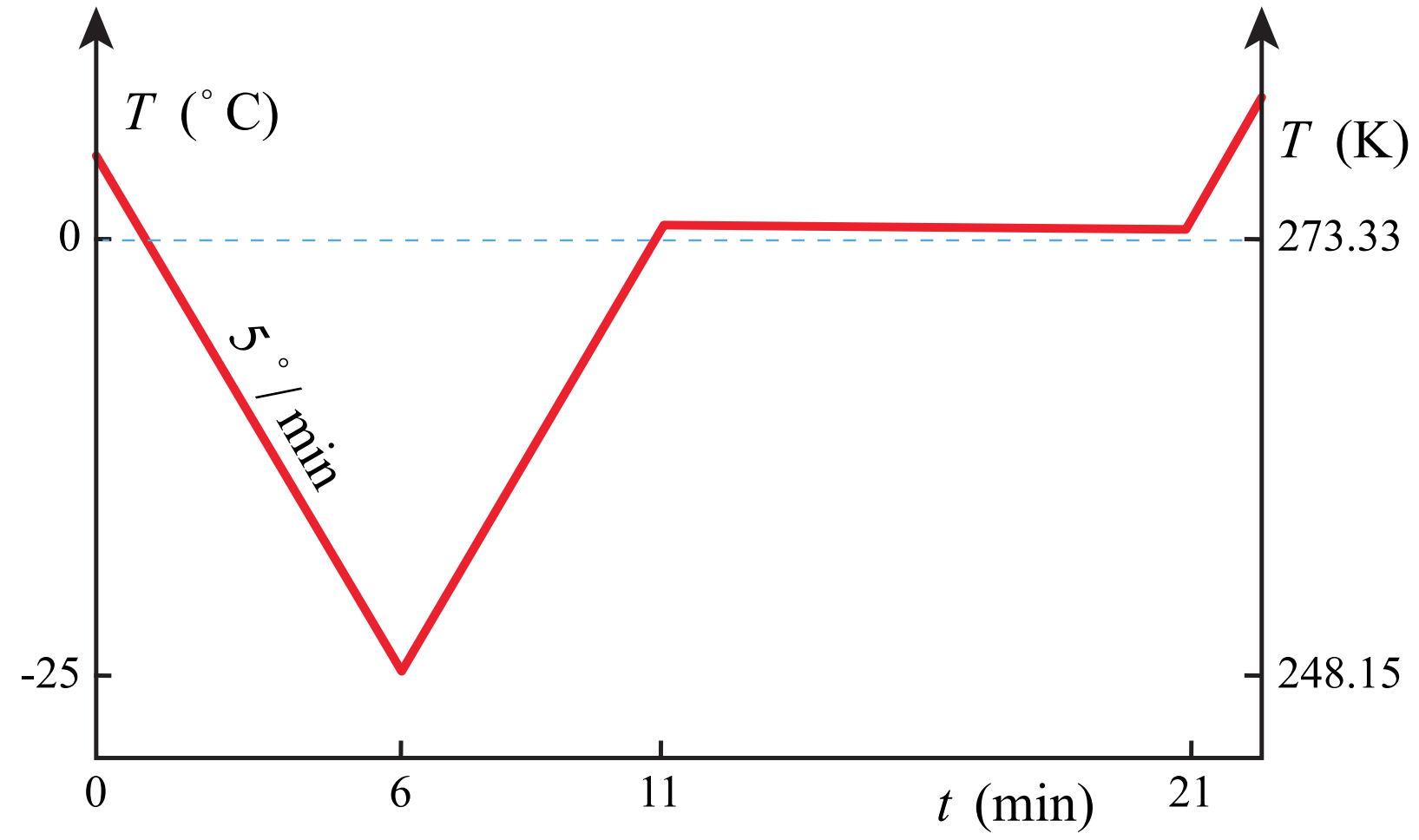

Figure 4: Schematic of the temperature sequence experienced by water drops in CP. Note the cooling/heating rate is constant $5^{\circ} / \mathrm{min}$ and the plateau is at $0.2^{\circ} \mathrm{C}(273.15 \mathrm{~K})$. 
follows: (i) the temperature, $\mathrm{T}$, decreases from $5^{\circ} \mathrm{C}(273 \mathrm{~K})$ to $-25^{\circ} \mathrm{C}(248 \mathrm{~K})$ at $5^{\circ} / \mathrm{min}$ to convert the water drop into ice; (ii) $T$ increases to $0.2^{\circ} \mathrm{C}(273.35 \mathrm{~K})$, again with a temperature ramp of $5^{\circ} / \mathrm{min}$; (iii) $\mathrm{T}$ remains constant at $0.2^{\circ} \mathrm{C}(273.35 \mathrm{~K})$ for 10 minutes, so that the ice melts and CP hydrates can grow; (iv) $T$ increases to room temperature and the system dissociates. Note the minimum reported CP hydrate equilibrium dissociation temperature vary from 7 to $7.7^{\circ} \mathrm{C}$ (280.15 to $280.85 \mathrm{~K}$ ) according to Zhang et al., ${ }^{4}$ Sakemoto et al., ${ }^{17}$ Sloan and Koh ${ }^{1}$ and Aman et al. ${ }^{16}$

\section{Results and discussions}
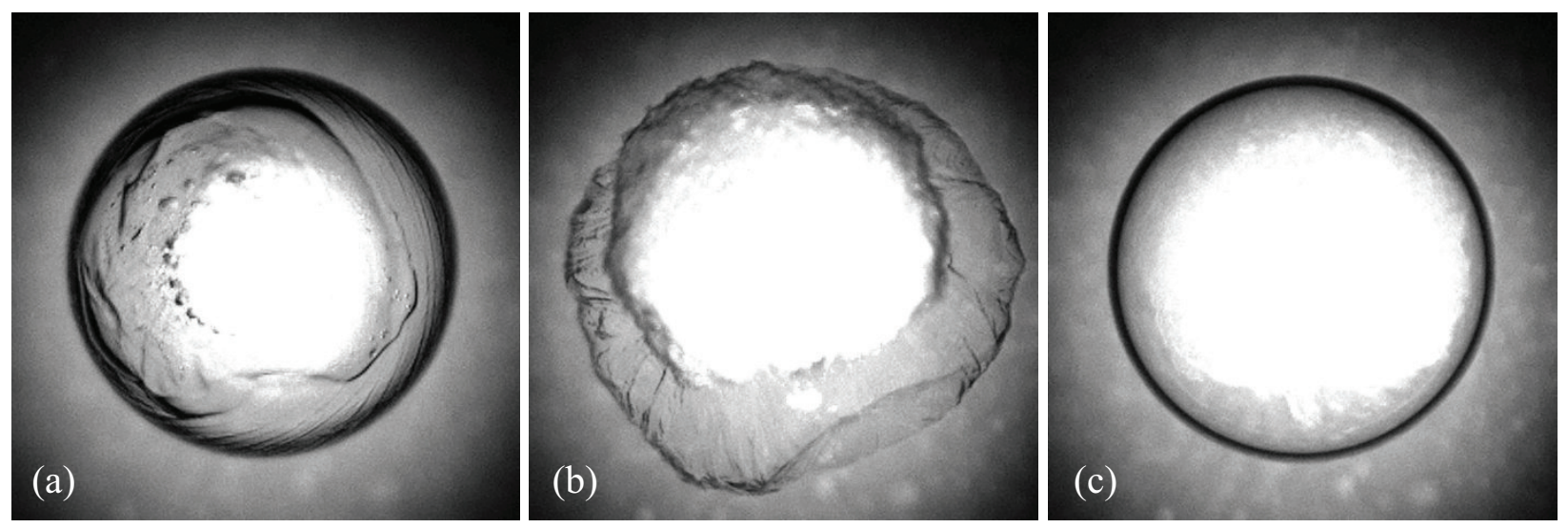

Figure 5: View from the top of the time evolution of a water drop in $\mathrm{CP}$ experiencing the temperature sequence from room temperature to $-25^{\circ} \mathrm{C}(248 \mathrm{~K})$, where crystallization occurs, (a), to $0.2^{\circ} \mathrm{C}(273.15 \mathrm{~K})$, where ice melts and hydrate grows, (b), and back to room temperature (c). The drop volume is $2 \mu \mathrm{l}$, which corresponds to about $1.5 \mathrm{~mm}$ in diameter.

The results consists in a series of pictures that report the morphology of water drops in $\mathrm{CP}$ and a table for the ice nucleation temperature, the duration of $\mathrm{CP}$ hydrate formation and the dissociation temperature.

\section{CP hydrates from ice}

Following the temperature sequence described earlier, the water drop reaches three steady states where it (i) freezes into ice balls, (ii) forms hydrates and (iii) dissociates to liquid 
drops. Figure 5 presents images of the three steady states. These are bright in the center because of transmitted light going through the center of the drop. The freezing takes place at $T$ between $-17.4^{\circ} \mathrm{C}(255.75 \mathrm{~K})$ and $-19.1^{\circ} \mathrm{C}(254.05 \mathrm{~K})$ and the time scale for complete crystallization is of the order of a few seconds. The critical freezing temperature is stochastic in nature. ${ }^{35}$ Moreover, the drop is frozen from the bottom, so the propagation of a freezing front and a reduction of mass density explains the pointy ice-drop with a sharp tip. ${ }^{36}$ This tip can be observed in figure $5(\mathrm{a})$.

During the second temperature ramp, the temperature reaches $0.2^{\circ} \mathrm{C}(273.35 \mathrm{~K})$ and the ice starts to melt. During the temperature plateau at $0.2^{\circ} \mathrm{C}(273.35 \mathrm{~K})$, the ice continues to melt and the hydrates grow. This process takes few minutes before reaching a steady state. The figure 5(b) presents an image of the steady state after the ice melted. Experiments ${ }^{37}$ on larger drops and gas hydrates (methane and carbon dioxide) at elevated pressures have shown long term hydrates growth (over hours). At least, our experiments have reached metastable states where lateral growth has taken place. Although our experiment only had access to the top view, the ice particle with growing hydrates seems to be expanded. This may be interpreted as porous shell hydrate formed. The full conversion of the ice into hydrate also depends on the shell material properties (porosity and stiffness), the conversion rate and the diffusion of water through the shell. For water drops in CP, the lateral growth velocity and interface thickness have been investigated before ${ }^{14,21,38}$ and some of the properties of the porous hydrate shell are available. The surface morphology of the hydrate sphere (figure $5(\mathrm{~b})$ ) differs from the ice ball (figure 5(a)), specifically the roughness. In an emulsion, the increase of roughness might lead to an increase in the apparent volume fraction of the disperse phase, hence a significant increase of the viscosity. The surface morphology is known to depends on the magnitude of the driving force, i.e. the sub-cooling temperature. ${ }^{20,21,37,38}$ Here the sub-cooling temperature is relatively high and about $7^{\circ} \mathrm{C}(280 \mathrm{~K})$. During the last temperature ramp, the temperature increases and the hydrate melts back to a water drop as depicted in figure $5(\mathrm{c})$. 

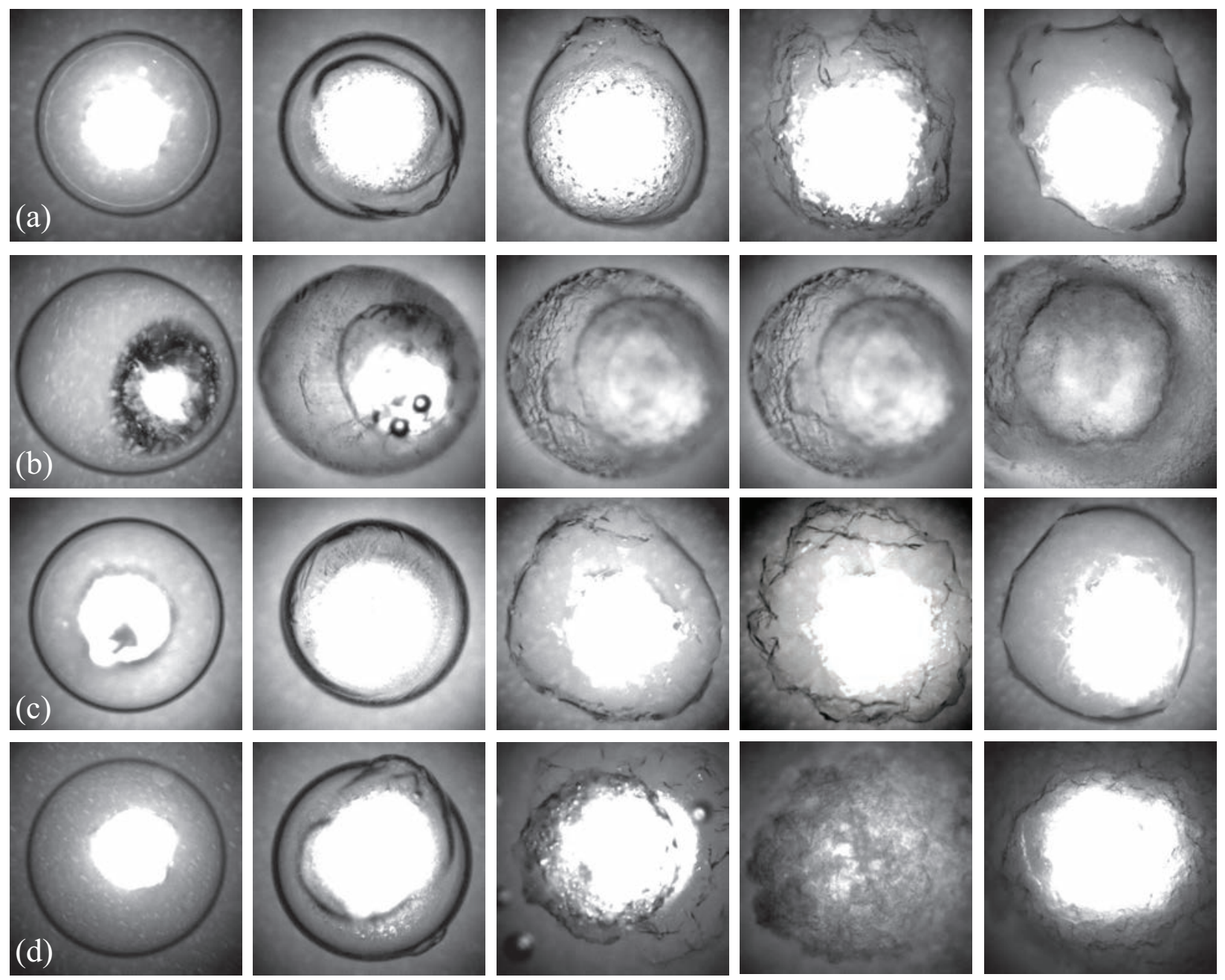

Figure 6: Snapshots of CP hydrate growth for various $C_{\mathrm{S} 80}$ : (a) $0 \%$, (b) $0.0001 \%$, (c) $0.001 \%$ and (d) $0.1 \%$, the only concentration above CMC. The first image of each line is the initial water drop with an initial volume of $2 \mu \mathrm{l}$, which corresponds to a diameter of about $1.5 \mathrm{~mm}$, at $T=5^{\circ} \mathrm{C}(278.15 \mathrm{~K})$. The images on the second, third and forth position are at $T=0.2^{\circ} \mathrm{C}$ $(280 \mathrm{~K})$. The last image of each line illustrates the onset of hydrate melting at a temperature around $7^{\circ} \mathrm{C}(273.35 \mathrm{~K})$. 


\section{Effect of Span 80 surfactant}

Table 1: Summary of the experiments for different CP-Span 80 solutions: interfacial tension at different temperatures, time for hydrate formation, $t_{f}$, at $0.2^{\circ} \mathrm{C}(273.35 \mathrm{~K})$, dissociation temperature, $T_{d}$, and time for dissociation, $t_{d}$.

\begin{tabular}{cccccc}
\hline$C_{\mathrm{S} 80}[\% \mathrm{wt}]$ & $\sigma_{3^{\circ} \mathrm{C}(275 \mathrm{~K})}[\mathrm{mN} / \mathrm{m}]$ & $\sigma_{20^{\circ} \mathrm{C}(293 \mathrm{~K})}[\mathrm{mN} / \mathrm{m}]$ & $t_{f}[\mathrm{~s}]$ & $T_{d}\left[{ }^{\circ} \mathrm{C}(\mathrm{K})\right]$ & $t_{d}[\mathrm{~s}]$ \\
\hline 0 & 51.3 & 49.3 & 257 & $6.7(279.85)$ & 27 \\
0.0001 & 45.7 & 45.2 & 457 & $5.7(278.85)$ & 37 \\
0.001 & 40.4 & 37.9 & 467 & $5.5(278.65)$ & 39 \\
0.1 & 5.1 & 4.3 & 341 & $7.7(280.85)$ & 135 \\
\hline
\end{tabular}

The temperature sequence has been applied to drops in various baths of $\mathrm{CP}$ and $\mathrm{CP}$ mixtures with Span 80 of different concentrations, $C_{\mathrm{S} 80}$. The morphology of the CP hydrates is tentatively related to the interfacial tension between water and CP, which was measured for different temperatures in figure 2. Specifically, the time evolution of the drop during the temperature cycle is presented for $C_{\mathrm{S} 80}=0,0.0001,0.001$ and $0.1 \%$ in figure $6(a),(b),(c)$ and $(d)$, respectively. Only the last concentration, figure $6(d)$, is above the CMC. The first image of the line is the initial water drop pinned to the hole of the PTFE disk. Note the slight differences in droplet area of the initial image, which is due to the effect of the hole used to prevent the drop to move: its shape can varie slightly and modify the position of the contact line and subsequently the droplet area. Moreover, the increase in surfactant concentration is know to flattens the droplet and induce an increase of the droplet area. The second, third and forth images render the hydrates growth. The last image of each line illustrates the melting of the hydrate. The lighting of each line is independent. The thinning and the roughening of the hydrate interface is clear, specially for $C_{\mathrm{S} 80}=0.0001 \%$ (figure $6(b)$ ), which corresponds to a concentration below CMC where the surfactant molecules are not enough to cover the whole drop. For $C_{\mathrm{S} 80}>\mathrm{CMC}$, presented in figure $6(d)$, the morphology of the hydrate seems to be slightly more dense, as the light is blocked. Yet, the detail roughness morphology of the porous hydrate seems to be similar and independent of the $C_{\mathrm{S} 80}$. In other words, the low interface tension and the associated energy cost of excess interfacial area seems negligible compared to the sub-cooling driving force, which favors hydrates formation. 


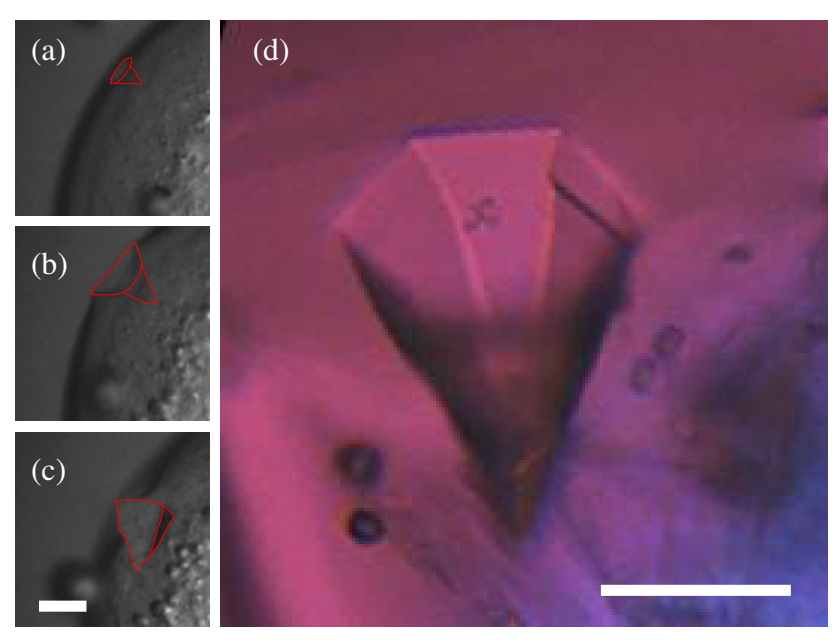

Figure 7: (a-c) Growth of a conical hydrate crystal in a solution of CP with $0.1 \%$ Span 80 over 22 seconds during the temperature plateau at $T=0.2^{\circ} \mathrm{C}(273.35 \mathrm{~K})$. The red outlines identifies typical hydrate crystals borders and the scale bar represents $100 \mu \mathrm{m}$. (d) View of the conical crystal through polarized light microscopy. The scale bar represents $25 \mu \mathrm{m}$.

During the hydrate growth, conical structures have been observed in the mixture of $0.1 \%$ Span 80 in CP. Figure 7 presents images of the growth of conical hydrates also observed in previous experimental studies. ${ }^{8,19}$ These structures can be seen to grow after 16 seconds through the temperature plateau at $T=0.2^{\circ} \mathrm{C}(273.35 \mathrm{~K})$. At 22 seconds, the crystals rise upwards, persist and aggregate to form bulk hydrate.

Table 1 summarizes the experiments on interfacial tension measurements at different temperature: $\sigma_{3{ }^{\circ} \mathrm{C}(275 \mathrm{~K})}$ and $\sigma_{20^{\circ} \mathrm{C}(293 \mathrm{~K})}$. In addition, the duration of the hydrate formation, $t_{f}$, when reaching the plateau temperature at $T=0.2^{\circ} \mathrm{C}(273.35 \mathrm{~K})$ is also reported. $t_{f}$ increases with $C_{\mathrm{S} 80}$. This is consistent with the effect of surfactants that will settle at the interface between water and $\mathrm{CP}$ and will prevent the motion of water molecules and CP molecules and the formation of hydrates structures. Table 1 also reports information about the dissociation temperature and duration, $T_{d}$ and $t_{d}$. The dissociation temperature remains in a range consistent with previous experiments that found $T_{d}$ from 7 to $7.7^{\circ} \mathrm{C}(280.15$ to $280.85 \mathrm{~K}$ ) according to Zhang et al., ${ }^{4}$ Sakemoto et al. ${ }^{17}$ Sloan and Koh ${ }^{1}$ and Aman et al. ${ }^{16}$ Moreover, the duration for total dissociation, $t_{d}$, increases with $C_{\mathrm{S} 80}$. This seems to be directly related to the drop in interfacial tension between water and $\mathrm{CP}$, which was measured 
for different temperatures in figure 2 .

\section{Conclusion}

Microscopic observations of the formation and the growth of cyclopentane (CP) hydrates crystals, at atmospheric pressure, were performed through temperature quenches of water drops. These observations were performed systematically using two microliters water drops in CP baths with a specific temperature protocol that nucleated ice at low temperature. The simultaneous melting of water and growth of hydrates are observed during a temperature plateau at $0.2^{\circ} \mathrm{C}(273.35 \mathrm{~K})$ for 10 minutes.

These morphology data are completed with detailed interfacial tension measurements for different temperature and surfactant concentration. A large concentration range of Span 80 surfactant was tested and it seems a slightly rougher porous shell is observed. In addition, the kinetic time scale is increased by the addition of surfactant. Future studies should be carried out with more realistic systems with several drops, different sizes of drops, different types of oils and surfactants in order to make the link between these fundamental experiments and field operations, specifically the adhesion forces and the relation with plugging of pipelines.

\section{Acknowledgement}

The authors thank Frédéric Renou and Michel Grisel for providing nitrogen. The present work benefited from the support from the French National Research Agency (ANR) through the program "Investissements d'Avenir" LabEx EMC 3 and the project BIOENGINE, which was co-financed by the European Union with the European regional development fund and by the Normandie Regional Council. VA is supported by a doctoral grant from the Région Normandie. 


\section{References}

(1) Sloan Jr, E. D.; Koh, C. Clathrate hydrates of natural gases; CRC press, 2007.

(2) Giavarini, C.; Hester, K. Gas hydrates: Immense energy potential and environmental challenges; Springer, 2011.

(3) Ribeiro, C. P.; Lage, P. L. C. Modelling of hydrate formation kinetics: State-of-the-art and future directions. Chem. Eng. Sci. 2008, 63, 2007-2034.

(4) Zhang, J.; Lee, J. Equilibrium of hydrogen+cyclopentane and carbon dioxide+cyclopentane binary hydrates. J. Chem. Eng. Data 2009, 54, 659-661.

(5) Camargo, R.; Palermo, T.; Sinquin, A.; Glenat, P. Rheological characterization of hydrate suspensions in oil dominated systems. Ann. N. Y. Acad. Sci. 2000, 912, 906916.

(6) Sinquin, A.; Palermo, T.; Peysson, Y. Rheological and flow properties of gas hydrate suspensions. Oil Gas Sci. Technol. 2004, 59, 41-57.

(7) Pauchard, V.; Darbouret, M.; Palermo, T.; Peytavy, J.-L. Gas hydrate slurry flow in a black oil. Prediction of gas hydrate particles agglomeration and linear pressure drop. 13th International Conference on Multiphase Production Technology. 2007.

(8) Zylyftari, G.; Ahuja, A.; Morris, J. F. Nucleation of cyclopentane hydrate by ice studied by morphology and rheology. Chem. Eng. Sci. 2014, 116, 497-507.

(9) Karanjkar, P. U.; Ahuja, A.; Zylyftari, G.; Lee, J. W.; Morris, J. F. Rheology of cyclopentane hydrate slurry in a model oil-continuous emulsion. Rheol. Acta 2016, 55, 235-243.

(10) Peixinho, J.; Karanjkar, P. U.; Lee, J. W.; Morris, J. F. Rheology of hydrate forming emulsions. Langmuir 2010, 26, 11699-11704. 
(11) Ahuja, A.; Zylyftari, G.; Morris, J. F. Yield stress measurements of cyclopentane hydrate slurry. J. Non-Newton. Fluid Mech. 2015, 220, 116-125.

(12) Zylyftari, G.; Lee, J. W.; Morris, J. F. Salt effects on thermodynamic and rheological properties of hydrate forming emulsions. Chem. Eng. Sci. 2013, 95, 148-160.

(13) Zylyftari, G.; Ahuja, A.; Morris, J. F. Modeling oilfield emulsions: comparison of cyclopentane hydrate and ice. Energy Fuels 2015, 29, 6286-6295.

(14) Karanjkar, P. U.; Lee, J. W.; Morris, J. F. Calorimetric investigation of cyclopentane hydrate formation in an emulsion. Chem. Eng. Sci. 2012, 68, 481-491.

(15) Fidel-Dufour, A.; Gruy, F.; Herri, J.-M. Rheology of methane hydrate slurries during their crystallization in a water in dodecane emulsion under flowing. Chem. Eng. Sci. 2006, 61, 505-515.

(16) Aman, Z. M.; Koh, C. A. Interfacial phenomena in gas hydrate systems. Chem. Soc. Rev. 2016, 45, 1678-1690.

(17) Sakemoto, R.; Sakamoto, H.; Shiraiwa, K.; Ohmura, R.; Uchida, T. Clathrate hydrate crystal growth at the seawater/hydrophobic-guest-liquid interface. Cryst. Growth Des. 2009, 10, 1296-1300.

(18) Ishida, Y.; Takahashi, Y.; Ohmura, R. Dynamic behavior of clathrate hydrate growth in gas/liquid/liquid system. Cryst. Growth Des. 2012, 12, 3271-3277.

(19) Karanjkar, P. U.; Lee, J. W.; Morris, J. F. Surfactant effects on hydrate crystallization at the water-oil interface: hollow-conical crystals. Cryst. Growth Des. 2012, 12, 38173824 .

(20) Mitarai, M.; Kishimoto, M.; Suh, D.; Ohmura, R. Surfactant effects on the crystal growth of clathrate hydrate at the interface of water and hydrophobic-guest liquid. Cryst. Growth Des. 2015, 15, 812-821. 
(21) de Baños, M. L. M.; Hobeika, N.; Bouriat, P.; Broseta, D.; Enciso, E.; Clément, F.; Brown, R. How do gas hydrates grow on a substrate? Cryst. Growth Des. 2016,

(22) Sonin, A. A.; Palermo, T.; Lubek, A. Effect of a dispersive surfactant additive on wetting and crystallisation in a system: water-oil-metal substrate. Application to gas hydrates. Chem. Eng. J. 1998, 69, 93-98.

(23) Ning, D.; Liu, X. Y. Controlled ice nucleation in microsized water droplet. Appl. Phys. Lett. 2002, 81, 445-447.

(24) Beltrán, J. G.; Servio, P. Morphological investigations of methane-hydrate films formed on a glass surface. Cryst. Growth Des. 2010, 10, 4339-4347.

(25) Rane, J. P.; Pauchard, V.; Couzis, A.; Banerjee, S. Interfacial rheology of asphaltenes at oil--water interfaces and interpretation of the equation of state. Langmuir 2013, 29, 4750-4759.

(26) Leopércio, B. C.; de Souza Mendes, P. R.; Fuller, G. G. Growth kinetics and mechanics of hydrate films by interfacial rheology. Langmuir 2016, 32, 4203-4209.

(27) Baek, S.; Min, J.; Lee, J. W. Equilibria of cyclopentane hydrates with varying HLB numbers of sorbitan monoesters in water-in-oil emulsions. Fluid Phase Equilibria 2016, 413, 41-47.

(28) Perry, R. H.; Green, D. W. Perry's chemical engineers' handbook; McGraw-Hill Professional, 1999.

(29) Kell, G. S. Density, thermal expansivity, and compressibility of liquid water from $0^{\circ}$ to $150^{\circ} \mathrm{C}$ : Correlations and tables for atmospheric pressure and saturation reviewed and expressed on 1968 temperature scale. J. Chem. Eng. Data 1975, 20, 97-105.

(30) Zhang, Y.; Debenedetti, P. G.; Prud'homme, R. K.; Pethica, B. A. Differential scanning 
calorimetry studies of clathrate hydrate formation. J. Phys. Chem. B 2004, 108, 1671716722.

(31) Song, B.; Springer, J. Determination of interfacial tension from the profile of a pendant drop using computer-aided image processing: 1. Theoretical. J. Colloid Interface Sci. 1996, 184, 64-76.

(32) Zeppieri, S.; Rodríguez, J.; López de Ramos, A. Interfacial tension of alkane + water systems. J. Chem. Eng. Data 2001, 46, 1086-1088.

(33) Pan, R.; Green, J.; Maldarelli, C. Theory and experiment on the measurement of kinetic rate constants for surfactant exchange at an air/water interface. J. Colloid Interface Sci. 1998, 205, 213-230.

(34) de Baños, M. L. M.; Carrier, O.; Bouriat, P.; Broseta, D. Droplet-based millifluidics as a new tool to investigate hydrate crystallization: Insights into the memory effect. Chem. Eng. Sci. 2015, 123, 564-572.

(35) Carte, A. E. The freezing of water droplets. Proc. Phys. Soc. B 1956, 69, 1028.

(36) Snoeijer, J. H.; Brunet, P. Pointy ice-drops: How water freezes into a singular shape. Am. J. Phys. 2012, 80, 764-771.

(37) Servio, P.; Englezos, P. Morphology of methane and carbon dioxide hydrates formed from water droplets. AIChE J. 2003, 49, 269-276.

(38) Taylor, C. J.; Miller, K. T.; Koh, C. A.; Sloan, E. D. Macroscopic investigation of hydrate film growth at the hydrocarbon/water interface. Chem. Eng. Sci. 2007, 62, $6524-6533$. 


\section{Graphical TOC Entry}

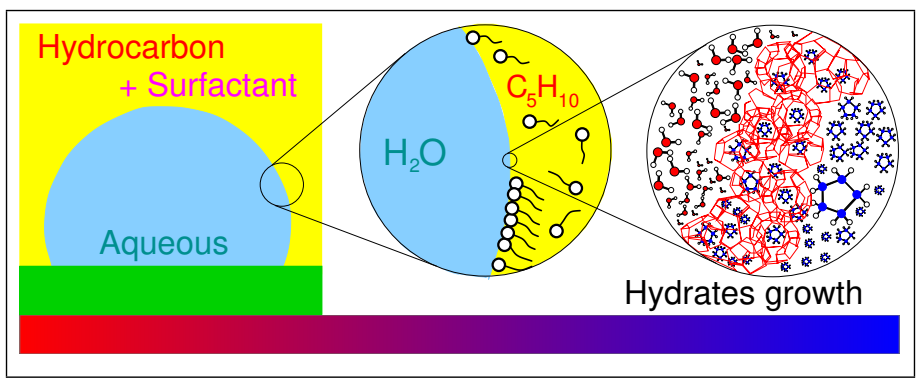

